\title{
Accounting for uncertainty in search operations using AUVs
}

\author{
Nuno Abreu ${ }^{12}$, Nuno Cruz ${ }^{12}$ and Aníbal Matos ${ }^{12}$ \\ ${ }^{1}$ INESC TEC, Campus da FEUP, Rua Dr. Roberto Frias, 378, 4200-465 Porto, Portugal \\ ${ }^{2}$ FEUP - DEEC, Rua Dr. Roberto Frias, s/n, 4200-465 Porto, Portugal \\ Emails: $\{$ nabreu, nacruz, anibal $\} @$ inesctec.pt
}

\begin{abstract}
Traditional coverage path planners create lawnmower-type paths in the operating area completely ignoring the uncertainty in the vehicle's position. However, in the presence of significant uncertainty in localization estimates, one can no longer guarantee that the vehicle will cover all the area according to plan. Aiming to bridge this gap, we present a coverage path planning technique for search operations which takes into account the vehicle's position and detection performance uncertainties and tries to minimize this uncertainty along the planned path. The objective is to plan paths, using a localization error model as input, to reduce as much uncertainty as possible and to minimize the extra path length (swath overlap) while satisfying mission feasibility constraints. We introduce an algorithm that calculates what will be the best moments for bringing the vehicle to surface to ensure a bounded position error. We also consider time and energy constraints that may influence the planned trajectory as path overlap is increased to account for uncertainty. Additionally we challenge the assumption frequently seen in coverage algorithms where two observations of the same target are considered independent.
\end{abstract}

Keywords - coverage, planning, localization, uncertainty, inertial navigation, AUV

\section{INTRODUCTION}

When covering an area using an autonomous underwater vehicle (AUV), it may not be possible to guarantee that all the area is covered since the path actually followed by the vehicle may deviate from the path planned by the coverage algorithm. The real path can deviate from the planned path due to:

- the existence of external disturbances such as sea currents and bad weather;

- the vehicle state estimate being uncertain and bounded or unbounded errors may exist;

- the performance of the motion controller not being good enough.

State estimation techniques commonly used in navigation systems, such as the Kalman filter, model sensor noise and produce estimates of the state prediction error. So a methodology is required to incorporate this knowledge of uncertainty (which is already available) in the planning stage, compensating for the limitations of the navigation system. Ignoring the uncertainty that accompanies probabilistic state estimates during planning can lead to a costly replanning stage where there is a need to cover all of the operating area (in the worst case) due to the existence of small gaps between parallel

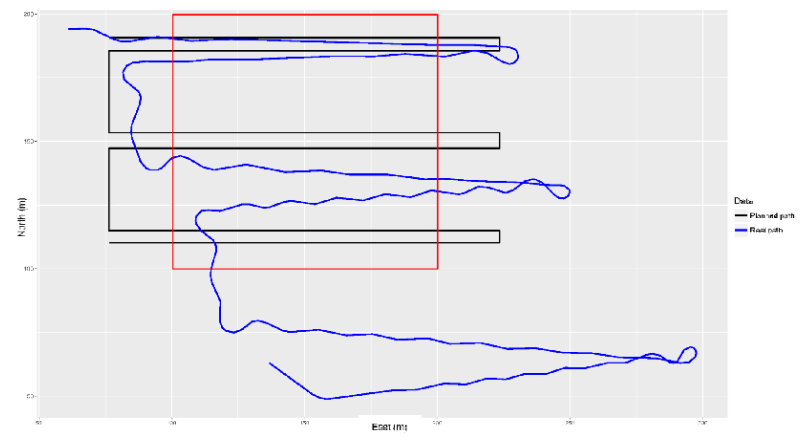

(a) Plot of the planned path (in black, planning area in red) and the actual path followed by the vehicle (in blue).

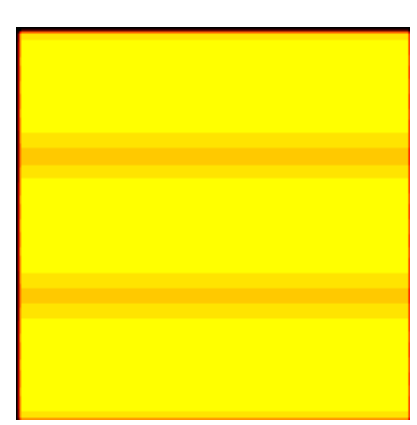

(b) Expected performance.

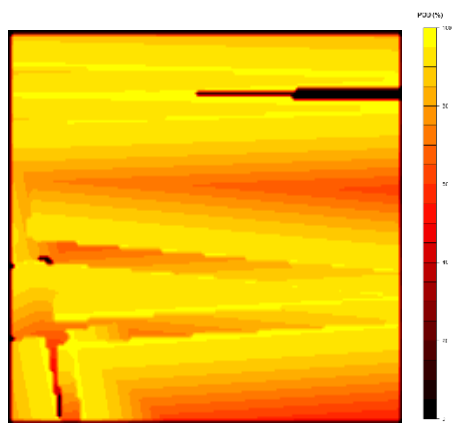

(c) Real performance.
Fig. 1. Effect of the navigation system's uncertainty in detection performance.

tracks where coverage performance is below what is required. Potentially, it can even lead to planning or even mission failure if the vehicle is not able to georeference acquired data due to the limited capability of smoothing algorithms to reduce past uncertainty.

Consider figure 1(a) which displays the outcome of a simulation where the AUV was trying to cover an area with a sidescan sonar. The performance of a low cost inertial measurement unit (IMU) with a high drifting bias is being simulated, chosen specifically for demonstrating the problem under study. Figure 1(b) shows the estimated performance for the planned path. Figure 1(c) shows the real performance given the position of the vehicle during the simulated mission. When determining the proper MCM coverage plan, one is usually constrained by the time to complete the operation and the risk to which vehicles and personnel may be subjected to. Thus, 
the scenario described above represents an unacceptable risk to units involved in an MCM operation and clearly needs to be accounted for.

An algorithm is needed to improve coverage performance, accounting for navigation errors along mission execution and adapting the planned path in response. There are several measures of mission performance, such as coverage factor, POD or probability of success (POS). Each of these measures can be considered appropriate for the application. In particular, the POD measure of performance is suggested (calculated using a lateral range curve as a function of range) together with the definition of a minimum level of performance at each point in the map. This would indirectly force complete coverage of the operating area while ensuring a lower bound on detection performance.

Aiming to bridge this gap, a CPP technique is presented which takes into account the robots motion and sensing uncertainty and tries to minimize this uncertainty along the planned path. The objective is to plan paths, using a state prediction error model as input, to reduce as much uncertainty as possible and to minimize the extra path length (swath overlap) while satisfying mission feasibility constraints. In the end a modified lawn-mower search pattern is produced to accomplish these objectives. In particular, the decay in detection performance over the discretized workspace is assessed by projecting the expected variation in range through the coverage sensor lateral range curve. An iterative algorithm based on simulated annealing is proposed to optimize track distance. The optimization algorithm simulates the uncertainty throughout the path and varies track spacing in order to ensure that the detection performance is above a given threshold. Additionally, whenever the uncertainty after a parallel track exceeds a user-provided threshold, a directive for getting a navigation update is inserted, seeking to reduce uncertainty and to avoid creating a mission plan that would take too long to execute due to the constant (growing) swath overlap.

\section{RELATED WORK}

Coverage path planning algorithms have considered the problem of localization uncertainty.

Colin et al. [1] present a "probably approximately correct measure of coverage performance, developed to account for sensor uncertainty. The authors argue that once vehicle localization error is significant, the definition of complete coverage must be adapted to become probabilistic. However, this approach considers an uniform sensor characteristic and provides no adaptive approach to account for the robot's pose uncertainty. Also, they assume a steady-state localization variance which is not a valid assumption for a vehicle using dead-reckoning or a inertial navigation system.

Hollinger el al. [3] presented an algorithm for accurate reconstruction of the seafloor that plans the AUV's dives seeking to maximize variance reduction along the planned path while also satisfying a budget constraint on maximum path length. They model the expected accuracy of the map using non-parametric Bayesian Regression in the form of
Gaussian Processes. The GP representation provides a measure of variance, which is used as a measure of uncertainty, as well as a mean value of altitude at each point of the bottom grid. Then they propose a greedy method to reduce this uncertainty by calculating efficient dive patterns: greedily select dives that maximize variance reduction until the budget is reached and then run a gradient optimization that perturbs each dive and locks the pattern into a local optimum. They estimate the variance reduction by first calculating the sum of variance in the area viewed by the dive.

Galceran et al. [4] present a survey path planning technique which takes into account the robots motion and sensing uncertainty and seeks to minimize this uncertainty along the planned path. They deal with the application constraints of surveying the target area in parallel tracks, at a certain constant altitude from the seafloor, and avoiding turns in order to maximize the quality of the sonar readings. A graph is built, representing the parallel tracks required to cover the target area. Then, a survey path is planned using two steps: first find the best possible order in which to cover the parallel track edges of the graph which minimizes the overall uncertainty along the path; second by inserting crossing track edges in the path found in the first step if, after tracing a parallel track, the uncertainty surpasses a given threshold. The robots position uncertainty along the determined path is estimated using a particle filter with the a priori bathymetry and simulated multibeam sonar measurements. Whenever the uncertainty after a parallel track exceeds a user-provided threshold, a crossing track through a salient area is inserted, seeking to reduce uncertainty. They are interested only in the uncertainty of the robots belief rather than in the position estimate. Their planning heuristic does not guarantee an optimal path with respect to uncertainty but it tackles the intractability of the planning problem by producing a low uncertainty solution, as demonstrated by their experimental results. On the other hand it can lead to lengthened paths due to the addition of multiple crossing tracks.

Paull et al. [5] presented a probabilistic framework within which paths can be generated that guarantee coverage based on new probabilistic coverage criteria. The pose belief is used to generate a coverage estimate that is maintained as the robot navigates around its workspace. This probabilistic representation is then used to adaptively plan paths for coverage based on an entropy reduction formulation.

We propose an approach similar to Paull et al. [5] but we introduce an algorithm that anticipates what will be the best moments for bringing the vehicle to surface to ensure a bounded position error. We also consider time and energy constraints that may influence the planned trajectory as path overlap is increased to account for uncertainty. Additionally we challenge the assumption frequently seen in coverage applications where two observations of the same target are considered independent. Modern research involving the calculation of the cumulative detection performance in different case studies assume complete independence, but there are some problems with this approach [2]. By performing cov- 


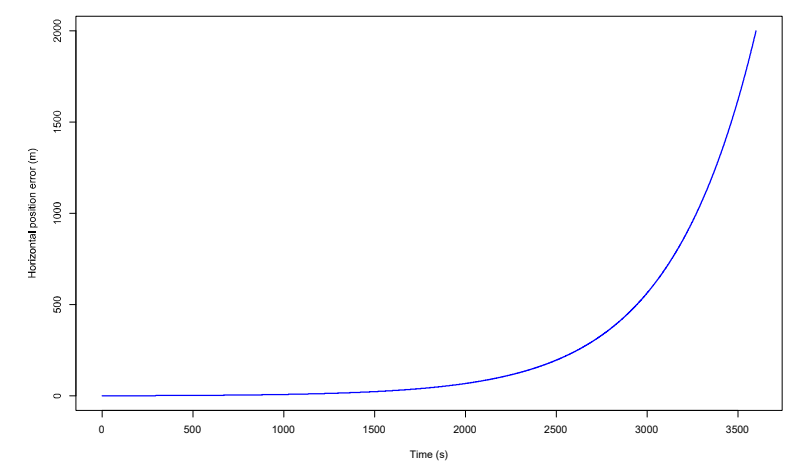

Fig. 2. Horizontal plane error model for a modern strap-down INS

erage on the same environment, with the same system and with increasing uncertainty in position it may be wise to consider that there is an indeterminate amount of correlation between observations. Therefore, we will present an alternative approach and assess the impact on detection performance estimation.

\section{NAVIGATION SYSTEMS UNCERTAINTY}

Accurate navigation information is essential for achieving the expected mission performance, for safe vehicle operation and recovery. For the data acquired by an AUV to be of value, the location from which the data has been acquired must be accurately known. Current practice in AUV navigation is focused on dead-reckoning, INS, acoustic systems and terrain aided techniques. Next, a brief description of the errors involved in these systems is given.

\subsection{Unbounded error}

The problem with relying on inertial navigation is that the position error increases without bound as the distance travelled by the vehicle increases. The rate of increase will be dependent on currents, the vehicle speed, the quality of dead reckoning algorithm and other sensor data. GPS can provide an accurate position update provided the vehicle is able to return to surface periodically for a position fix. The maximum vehicle travel time between surfacings for a position update will be determined by the navigation system's accuracy, as described in figure 2. Systems integrating a poor quality IMU will face an unacceptably high frequency of surfacing. Surfacing always comes with a cost, especially on extra mission execution time. For deep water applications, the time and energy needed by an AUV for transiting to the surface can be very unfavourable. Also, vehicles operating close to important shipping routes are in considerable danger of collision with surface vessels if they need to frequently return to surface for position fixes. Position drift rates for high quality IMU units (unaided INS) are on the order of several kilometres per hour. Integrating a DVL sensor in the INS can greatly improve performance with drift rates ranging from $0.01 \%$ to $0.1 \%$ of the distance travelled depending on the format of paths.

\subsection{Bounded error}

Acoustic transponders can be used as beacons to guide the AUV without the need for resurfacing. Long baseline (LBL) navigation systems provide position accuracy of a few meters with a maximum range on the order of a few kilometres. Errors in acoustic systems come from several sources. The main sources of error are: errors in the assumed array geometry and errors in the assumed sound speed profile. Positioning error comes from assuming incorrect positions of the array beacons. Reflection or multipath errors will result in erroneous timeof-flight values and thus wrong position fixes. Typically, LBL works well in deep water. When operating in shallower water, complex propagation effects become relevant and increase the frequency of bad position fixes. Even if the sound speed profile is known at the start of an AUV mission, the acoustic propagation environment can change during the mission (due to thermoclines, etc).

\section{Methodology}

Aiming to bridge this gap, a CPP technique is presented which takes into account the robots motion and sensing uncertainty and tries to minimize this uncertainty along the planned path. The objective is to plan paths, using a state prediction error model as input, to reduce as much uncertainty as possible and to minimize the extra path length (swath overlap) while satisfying mission feasibility constraints. Here a modified lawn-mower search pattern is used to accomplish these objectives. In particular, the decay in detection performance over the discretized workspace is assessed by projecting the expected variation in range through the coverage sensor lateral range curve. An iterative algorithm based on simulated annealing is also proposed to optimize track distance. This is an optimization algorithm that involves the simulation of uncertainty throughout the path and the implementation of a feedback policy that ensures that the detection performance will above a certain threshold. This approach has the distinct advantage that it can be easily added to existent coverage path planning algorithms as an add-on, optimizing existent plans before creating the mission directives.

\subsection{Uncertainty estimation}

The uncertainty in the state estimate provided by the AUVs navigation system when performing a coverage mission may cause the vehicle to deviate from the planned trajectory. This means that when traversing straight parallel transects, the vehicle may actually be closer or further away from a given point located between two transects, as depicted in figure 3.

The component of uncertainty in position, perpendicular to the transects, can be interpreted as the uncertainty on effective distance to that fixed point on the workspace. Thus, range is a random variable $R$. This variation in range to a given cell will result in a variation in detection performance. This means that detection performance on each cell of the workspace will now be a random variable $Y$ with a given probability distribution function (PDF) instead of a fixed quantity. It is 


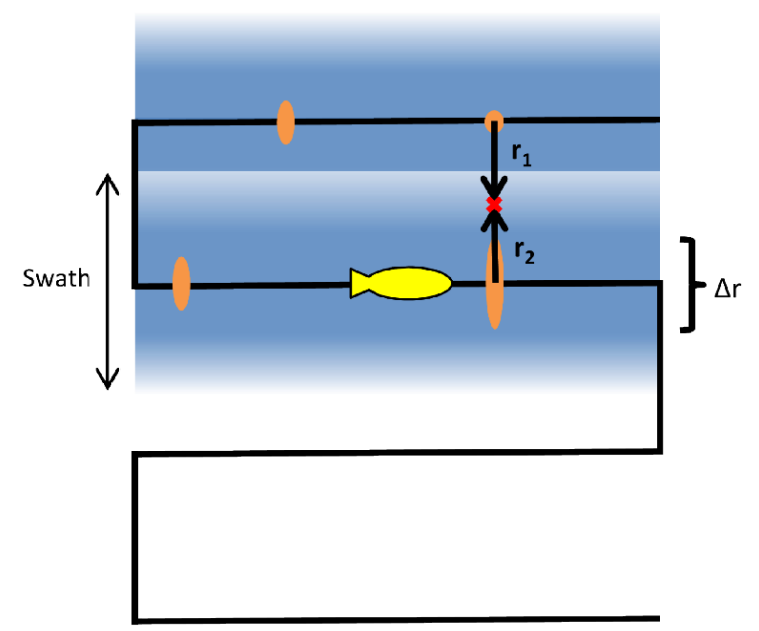

Fig. 3. Example of a lawnmower trajectory followed by the AUV. As the vehicle travels along the path, the uncertainty of the navigation systems estimates (in orange) increases. The distance to a fixed point (in red) cannot be considered static, from a planning perspective, given the uncertainty in position.

assumed that the PDF of $R, f_{R}$, can be approximated by a Gaussian function:

$$
f_{R}(r)=\frac{1}{\sqrt{2 \pi \sigma_{R}}} e^{-\frac{\left(x-\mu_{R}\right)^{2}}{2 \sigma_{R}^{2}}}
$$

Then the PDF of $Y, f_{Y}$, is obtained by applying the transformation technique for continuous random variables [6]:

$$
f_{Y}(y)=\left|\left(\frac{d}{d y} l^{-1}(y)\right)\right| f_{R}\left(l^{-1}(y)\right)
$$

where $l(r)$, which is the LRC function used to characterize the detection systems performance, is a one-to-one function and $l^{-1}(y)$ is its inverse. For this study, the inverse cube model is considered an appropriate function to represent the LRC and apply this methodology:

$$
l(r)=1-e^{-\frac{c}{r^{2}}}
$$

where $c$ is a constant that depends on the environment where the vehicle is operating and its velocity. By applying this principle, it is possible to derive the PDF and the CDF of $Y$, respectively:

$$
\begin{aligned}
f_{Y}(y)= & \frac{\sqrt{c}}{2(y-1)(\log (1-y))^{2} \sqrt{\frac{-1}{\log (1-y)}}} \mid \\
& \frac{1}{\sqrt{2 \pi \sigma_{R}}} e^{\frac{-\left(\sqrt{\frac{-c}{\log (1-y)}}-\mu_{R}\right)^{2}}{2 \sigma_{R}^{2}}}
\end{aligned}
$$

$$
F_{Y}(y)=\frac{\sigma_{R}\left|\frac{1}{\sqrt{\sigma_{R}}}\right| \operatorname{erf}\left(\frac{\sqrt{c} \sqrt{-\frac{1}{\ln (1-y)}}-\mu_{R}}{\sqrt{2} \sigma_{R}}\right)(y-1)}{2\left|\frac{1}{\sqrt{-\frac{1}{\ln (1-y)}}}\right| \sqrt{-\frac{1}{\ln (1-y)}}|y-1|}
$$

This was the process for estimating the detection performance distribution due to a single sonar measurement. As the vehicle may cover the same area twice with the sonar by traversing consecutive path transects, individual measurements need to be combined. For instance, in minehunting operations, where considerable risk is involved, the cost of overestimating the detection performance may be much higher than the cost of underestimating it. Hence, it should be beneficial to opt for a conservative estimate. Conservative estimates are obtained by assuming the existence of complete correlation, thus by choosing the higher of the individual estimates ( $\max$ operator). When combining the observations, one can assume dependence or independence, being the latter frequently chosen as it simplifies the procedure of calculating a PDF. Both approaches will be compared by also estimating a PDF assuming dependence and assessing the impact on detection performance estimation.

The combined detection performance of two measurements $Y_{1}$ and $Y_{2}$ is given by:

$$
Z=\max \left(Y_{1}, Y_{2}\right)
$$

For two independent random variables $Y_{1}$ and $Y_{2}, Z \mathrm{~s} \mathrm{CDF}$ and PDF can be calculated with the following expression, respectively [7]:

$$
\begin{gathered}
F_{Z}(z)=F_{Y_{1}}(z) F_{Y_{2}}(z) \\
f_{Z}(z)=f_{Y_{1}}(z) F_{Y_{2}}(z)+f_{Y_{2}}(z) F_{Y_{1}}(z)
\end{gathered}
$$

In a real world situation, it is difficult to assess the degree of correlation between the two observations. If such correlation exists, the true distribution for the combined detection performance $Z$ should be derived, if possible. However, due to the complexity of the mathematical functions, derivation of the equation is difficult. In such cases, Monte Carlo simulation is a suitable tool to estimate the real distribution function of $Z$ and, for this reason, it was applied here. Figure 4 presents a visual comparison between both approaches. It is possible to see that the first approach is a little conservative until halfway through the mission, as expected. But surprisingly, from there onwards, the first approach became increasingly pessimistic as time, and localization uncertainty as a consequence, increased. Such a performance supports the argument that one cannot just assume independence between observations without assessing the true relationship between the variables.

In our case, if the mission planner considered independence blindingly, then it would bring the parallel tracks excessively together to compensate for the growing uncertainty and that would increase mission time and energy expenditure beyond 


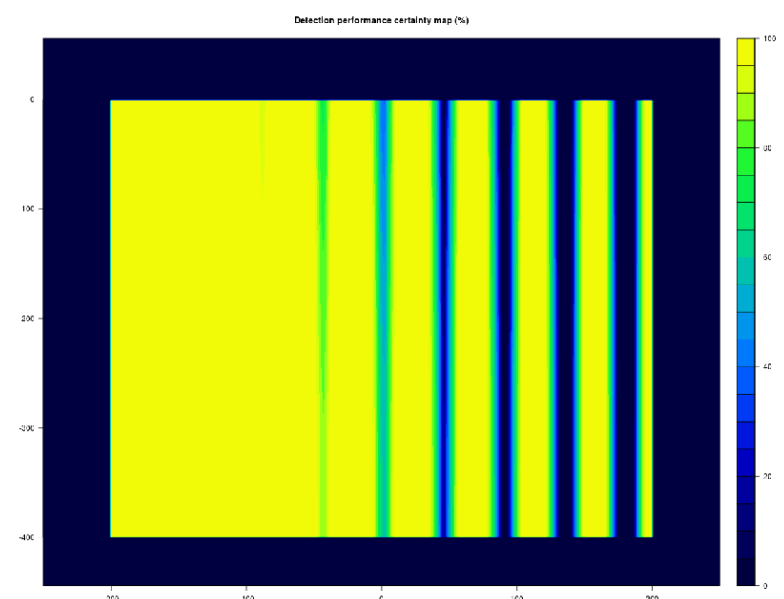

(a)

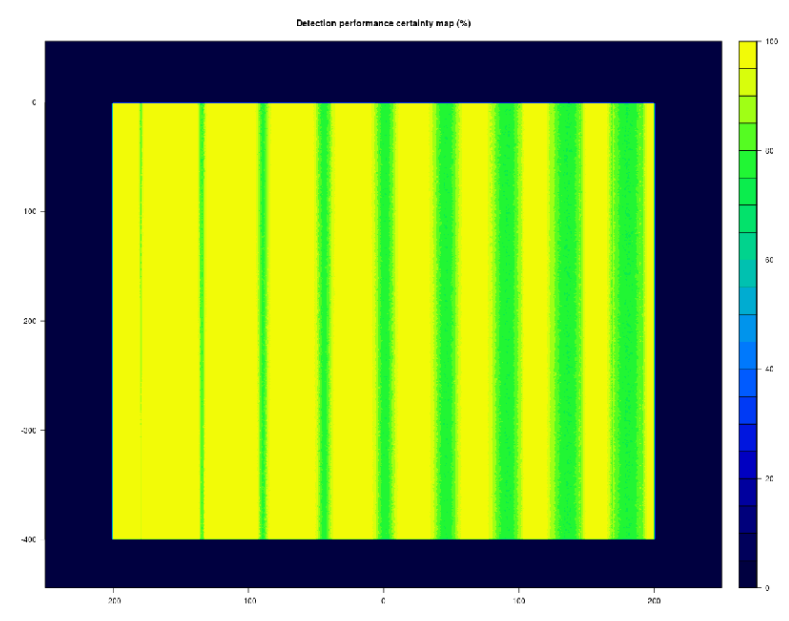

(b)

Fig. 4. Detection performance certainty maps considering (a) independence and (b) dependence between observations.

what was really required. Unfortunately, given the difficulty in deriving the real $F_{Z}$ and the time needed to perform the Monte Carlo simulation, there is no alternative to considering independence between the observations. To mitigate the effect on the calculation of the probabilistic measure of detection performance, an upper bound on time between surfacing manoeuvres is established to reduce localization uncertainty. In this experiment, assuming a navigation error model as depicted in figure 2 and by analysing the maps on figure 4 , a time interval of 575 seconds was chosen as the difference between the approaches was negligible up to this point.

\subsection{Uncertainty reduction procedure}

As a means to minimize the number of gaps in coverage resultant from uncertainty in the states estimates, our local optimization algorithm will adjust the position of the parallel transects in order to guarantee that the probability of the detection performance $Z$ being above a minimum value $D_{\min }$ is close to a predefined certainty level $C$ :
Consider the following assumptions:

- operating area $A$;

- $N$ tracks, parallel in projection to horizontal plane;

- each track $i$ marks the end of a subarea $a_{i}$, so there area $N+1$ subareas;

- a variance threshold, $V_{\max }$, that represents maximum variance allowed at any time during the mission;

- a maximum overlap parameter, $O_{\max }$, that represents the maximum overlap allowed between two consecutive swaths.

The inputs for this procedure are:

- lawnmower search pattern;

- topography map;

- navigation systems error function;

- LRC;

- minimum detection performance $D_{\min }$ and required certainty level $C$;

- available time and energy for executing the mission;

- $V_{\max }$;

- $O_{\max }$.

Then the outputs will be:

- optimized lawnmower search pattern;

- detection performance map;

- best time instants for surfacing for uncertainty reduction (if using INS).

A high level description of the algorithm is presented in the flowchart depicted on figure 5. The first phase consists in analysing variance growth along the trajectory. Each time the variance surpasses $V_{\max }$ (which is the maximum squared deviation determined previously) a surfacing request is added to reduce uncertainty, preferably at the start of the track. Since variance is a function of time and the velocity of the vehicle is known, the distance between each of these points can be determined.

Next is the spacing optimization procedure. In order to increase the certainty that the detection performance requirements will be met, track spacing needs to be reduced. Here, the maximum overlap between two tracks needs to be considered. This was introduced to avoid creating paths that are too long. When energy and time constraints are broken after adding extra tracks, this parameter will be reduced in order to create smaller paths. Reducing overlap may also lead to the performance requirements not being met, in which case the solution which offers the best possible $P\left(Z \geq D_{\min }\right)$ throughout all operating area is returned.

Simulated Annealing (SA) gives us the opportunity to quickly improve any solutions that are being considered. This algorithm has been designed to optimize two distinct objectives, namely:

- Minimize the uncovered area;

- Maximize $P\left(Z \geq D_{\text {min }}\right)$.

These objectives are prioritized differently according to our specific mission planning goals: the $P\left(Z \geq D_{\min }\right)$ can only be increased if there are no more uncovered areas. Note that decreasing the amount of uncovered area will lead to an 
increase in $P\left(Z \geq D_{\min }\right)$ but not to its maximization. This is a combinatorial problem where the best set of inter-track distances needs to be chosen, minimizing the uncertainty in detection performance over an area characterized by a specific topography. Distinct strategies are used depending on the type of sonar that is being used for seafloor mapping, more specifically if using multibeam (no nadir gap) or sidescan sonar (with nadir gap).

\section{A. Strategy for the case without nadir gap:}

\section{Initialization:}

- Determine the original spacing between tracks (kept constant by the EA);

- Neighbourhood size is set to the initial spacing.

\section{Evaluation:}

- Determine the amount of uncovered area and the $P(Z \geq$ $\left.D_{\min }\right)$ (per subarea and total)

- Accept the solution if better then the last or apply the Metropolis criterion [8].

\section{Check for termination:}

- Terminate when the temperature reaches a minimum value, when it converges or when a maximum number of iterations is achieved.

\section{Mutation:}

- Select the subarea between a pair of tracks with lower coverage quality;

- Place two tracks closer together, achieving higher coverage in the subarea in between;

- The amplitude of the mutation $\Delta_{i}$ is obtained from equation 10, where $r$ is a random number between -1 and $1, N$ is the neighbourhood size and $T$ is the temperature of the process. It must be inferior to the previous track spacing $s_{i}$ in subarea $i$;

- Update spacing $s_{i}$ (equation 9);

- Calculate the contribution (weights $w_{j}$ ) of each subarea $\Delta_{j}(j \neq i)$ to compensate for $\Delta_{i}$ (equations 14 and 12);

- If there are no uncovered areas (ucells $=0$ ) then maximize $P\left(Z \geq D_{\text {min }}\right)$ using a similar procedure where track spacing is decreased in the region where the uncertainty is higher; in this case the contribution of each region $\Delta_{j}$ depends directly on the uncertainty in each of those regions (equation 13).

$$
\begin{gathered}
s_{i}=s_{i}-\Delta_{i} \\
\Delta_{i}=r N T \\
\Delta_{i}=-\sum_{\substack{j=1 \\
j \neq i}}^{k} \Delta_{j} \\
w_{j}^{\prime}=\frac{1}{\text { ucells }_{j}} \\
w_{j}^{\prime}=\text { avgpod }_{j}
\end{gathered}
$$

$$
\begin{gathered}
w_{j}=\frac{w_{j}^{\prime}}{\sum_{\substack{j=1 \\
j \neq i}}^{k} w_{j}^{\prime}} \\
\Delta_{j}=w_{j} \times \Delta_{i}
\end{gathered}
$$

B. Strategy for the case with nadir gap:

The most used technique to handle this problem is the uneven lawn-mowing coverage pattern, where consecutive pairs of tracks cover each other's nadir. Here, the distinction between odd and even areas is performed because they must be handled differently. Even areas will have smaller spacing since the adjacent tracks need to be closer together in order to cover their nadir gaps. Even areas contain the complete nadir region belonging to each of the adjacent tracks, even the portion that lies on the adjacent areas (the nadir extends to each side of the track considering that the sonar is being carried with zero roll angle). This strategy differs from the previous one on the mutation phase:

\section{Mutation:}

- Similar to the previous one, except that adjusting odd subareas only affects other odd subareas, while adjusting even subareas only affects adjacent subareas;

- The purpose of controlling track spacing in even subareas is mainly to cover their nadir gaps, therefore only the adjacent subareas should compensate for the variation of track spacing;

- Even subareas should remain untouched when adjusting odd subareas so the nadir gaps remain covered.

\subsection{Determination of surfacing manoeuvres positions}

Additionally, the location where each surfacing manoeuvre will take place can also be optimized. Since smoothing algorithms have limited performance and the error grows exponentially, it is beneficial to redistribute these locations in order to keep the error as low as possible at any time during the mission. Originally, the locations of the surfacing manoeuvres are calculated every time the error is near its maximum value. It is not ideal if, for example, there is a surfacing manoeuvre near the end of the mission. In such cases, it is appropriate to redistribute the locations so the average error is lower. We assume that the number of manoeuvres is known, given that we know the total trajectory length, the vehicle velocity and the maximum amount of time between manoeuvres to keep a bounded error. Then, knowing the amount of manoeuvres and the total trajectory length, the ideal distance between the manoeuvres can be determined. Our algorithm tries to place the surfacing locations as evenly distributed as possible, near this ideal distance. Since these locations need to match a vertex in the trajectory it may be difficult to ensure that the real distances between surfacing manoeuvres are equal.

\section{DEMONSTRATION}

For demonstrating our uncertainty reduction approach, consider figure 6. Figure 6(a) shows the uncertainty map for the original trajectory. After executing the algorithm, an optimized 


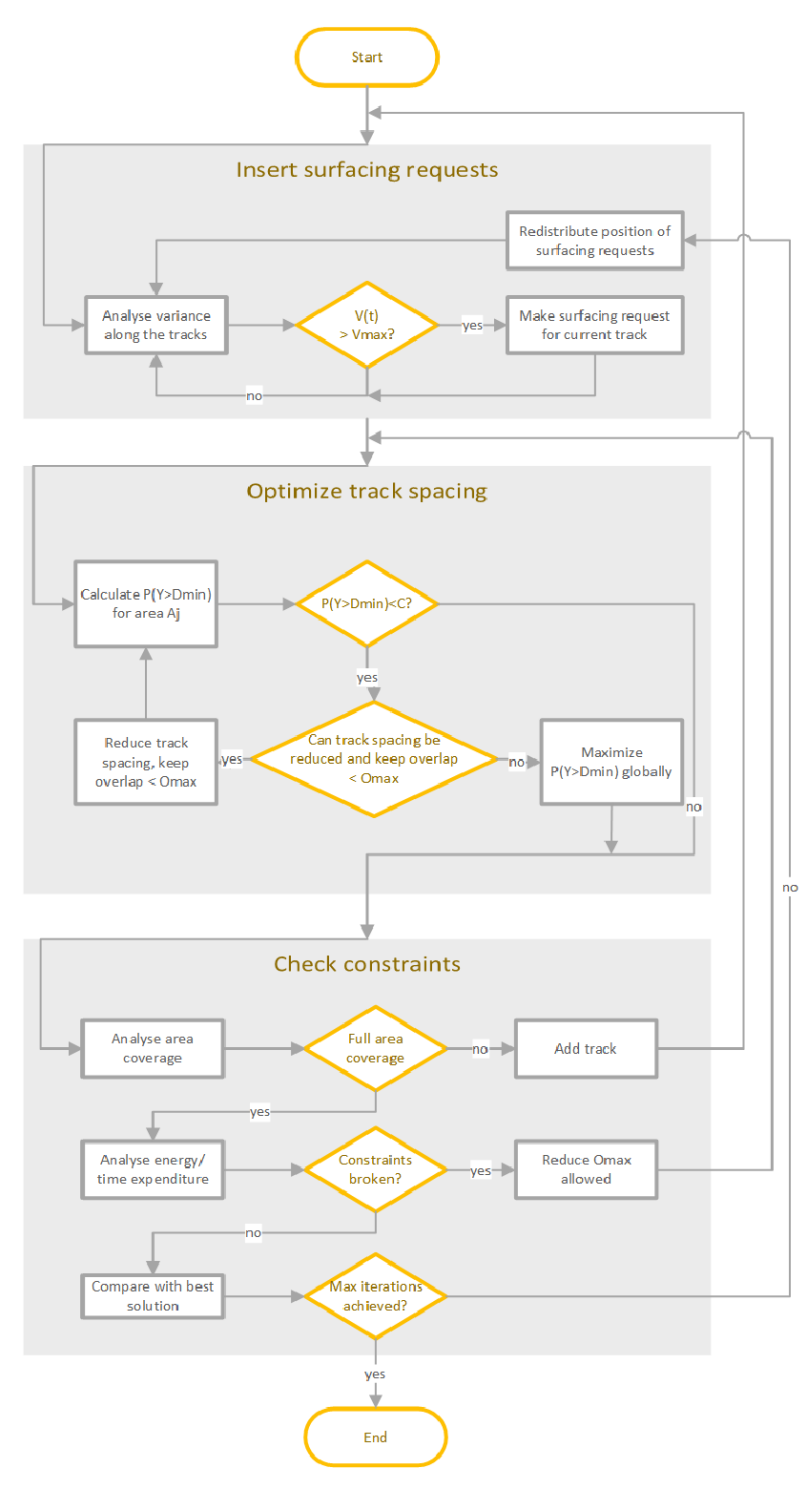

Fig. 5. Flowchart for the uncertainty reduction algorithm.

trajectory is obtained, represented in 6(b). Note that all nonyellow parts ceased to exist, meaning that the uncertainty was successfully reduced, as expected. The descriptive statistics for both cases are presented in tables 1 and 2 . In this scenario no additional tracks were added, but that may not always be the case. As explained earlier, we were quite conservative on the maximum amount of localization uncertainty which is tolerated and this is limiting the amount of overlap between swaths.

As expected, the redistribution of the surfacing locations also proved to be beneficial, as displayed on figure 7 . The estimated average localization error was reduced from 2.55 to 2.20 , although it was not possible to keep the surfacing locations as evenly distributed as desired.

\section{CONCLUSION}

In this paper a CPP technique for search operations was described which takes into account the vehicle's position and

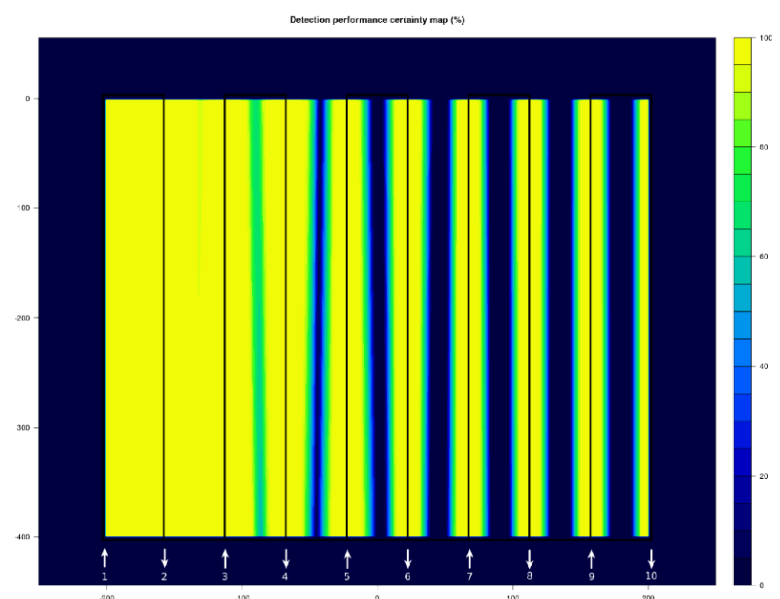

(a) Uncertainty for the original trajectory.

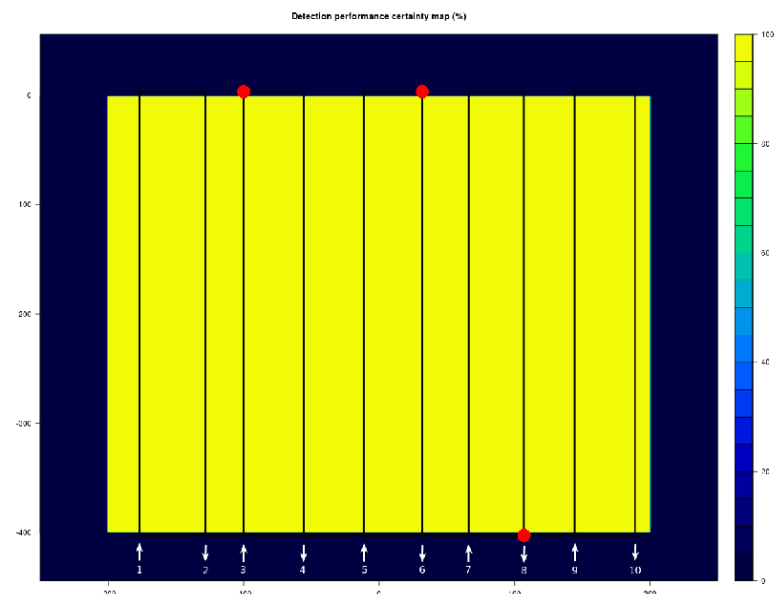

(b) Uncertainty for the optimized trajectory, considering surfacing maneuvers (red dots).

Fig. 6. Uncertainty reduction demonstration.

\section{TABLE 1}

INITIAL DESCRIPTIVE STATISTICS, BEFORE TRAJECTORY OPTIMIZATION. THE AVERAGE $P\left(Z \geq D_{\text {min }}\right)$ OVER THE OPERATING AREA WAS $72.2 \%$ AND THE AMOUNT OF CELLS Where $P\left(Z \geq D_{\min }\right)<C$ (MinRatio) WAS $45.3 \%$

\begin{tabular}{llll}
\hline Subarea & $P\left(Z \geq D_{\min }\right)$ & MinRatio & Spacing \\
\hline 1 & 0.000 & 0.000000 & 1.040 \\
\hline 2 & 99.750 & 0.000268 & 45.000 \\
\hline 3 & 99.131 & 0.003722 & 45.000 \\
\hline 4 & 92.787 & 0.034320 & 45.000 \\
\hline 5 & 78.769 & 0.054009 & 45.000 \\
\hline 6 & 65.493 & 0.065742 & 45.000 \\
\hline 7 & 57.582 & 0.070966 & 45.000 \\
\hline 8 & 54.048 & 0.072419 & 45.000 \\
\hline 9 & 52.527 & 0.074601 & 45.000 \\
\hline 10 & 49.613 & 0.077076 & 45.000 \\
\hline 11 & 0.000 & 0.000000 & 1.040 \\
\hline
\end{tabular}




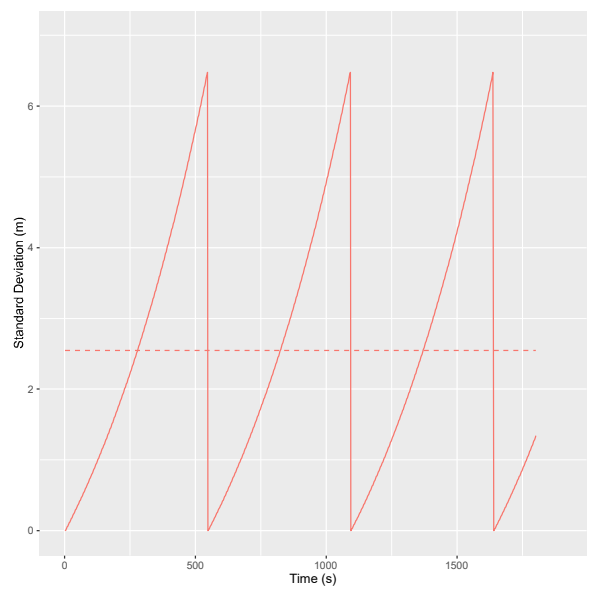

(a) Assuming initial surfacing locations calculated using a greedy algorithm.

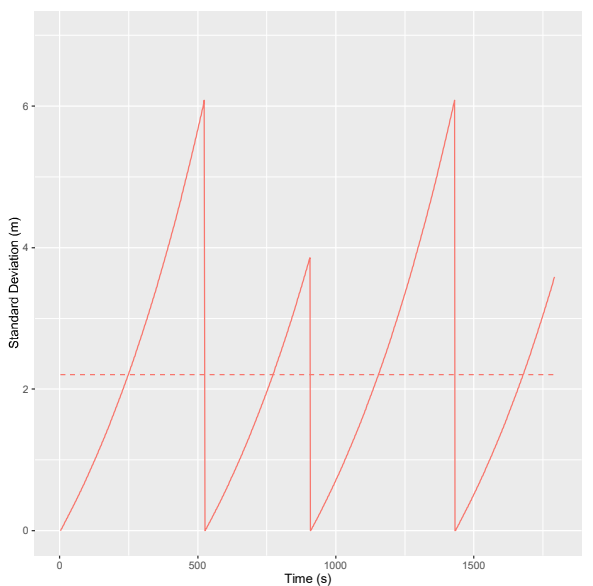

(b) Assuming optimized surfacing locations.

Fig. 7. Estimated localization error during the mission, considering the required surfacing manoeuvres for uncertainty reduction.

TABLE 2

FINAL DESCRIPTIVE STATISTICS, AFTER TRAJECTORY OPTIMIZATION. The AVERAGE $P\left(Z \geq D_{\text {min }}\right)$ OVER THE OPERATING AREA WAS $99.7 \%$ And The amount of Cells Where $P\left(Z \geq D_{\min }\right)<C$ (MinRatio) WAS $0.25 \%$.

\begin{tabular}{llll}
\hline Subarea & $P\left(Z \geq D_{\min }\right)$ & MinRatio & Spacing \\
\hline 1 & 99.748 & 0.000150 & 26.962 \\
\hline 2 & 99.712 & 0.000305 & 48.588 \\
\hline 3 & 99.750 & 0.000175 & 28.160 \\
\hline 4 & 99.647 & 0.000274 & 44.376 \\
\hline 5 & 99.749 & 0.000274 & 44.220 \\
\hline 6 & 99.750 & 0.000274 & 43.355 \\
\hline 7 & 99.750 & 0.000212 & 34.470 \\
\hline 8 & 99.713 & 0.000256 & 40.554 \\
\hline 9 & 99.750 & 0.000231 & 37.238 \\
\hline 10 & 99.749 & 0.000281 & 44.806 \\
\hline 11 & 99.750 & 0.000069 & 14.351 \\
\hline
\end{tabular}

detection performance uncertainties during mission execution and tries to minimize this uncertainty along the planned path. Such a technique is required in order to incorporate this knowledge of uncertainty in the planning stage, compensating for the limitations of the navigation system. Accounting for uncertainty during planning reduces the need for a costly replanning stage, where full area coverage may be required (in the worst case) due to the existence of small gaps in coverage between the parallel tracks, and increases the quality of the acquired data. Results showed that the method was successful in minimizing uncertainty and selecting the best positions for performing the surfacing manoeuvres.

\section{ACKNOWLEDGEMENT}

This work is financed by the ERDF European Regional Development Fund through the Operational Programme for Competitiveness and Internationalisation - COMPETE 2020 Programme within project POCI-01-0145-FEDER-006961, and by National Funds through the FCT Fundação para a Ciência e a Tecnologia (Portuguese Foundation for Science and Technology) as part of project UID/EEA/50014/2013.

\section{REFERENCES}

[1] Das, C., Becker, A., and Bretl, T., Probably approximately correct coverage for robots with uncertainty 2011 IEEE/RSJ International Conference on Intelligent Robots and Systems. IEEE, 2011.

[2] Carnes, J., Effective Area Searching for Ground Team Members: POD, Critical Spacing, and Smart Search Techniques, California OES and MRA report, 1993

[3] Hollinger, G., Urbashi Mitra, and Gaurav S. Sukhatme., Active and adaptive dive planning for dense bathymetric mapping, Experimental Robotics. Springer International Publishing, 2013.

[4] Galceran, E., et al., Uncertainty-driven survey path planning for bathymetric mapping, 2013 IEEE/RSJ International Conference on Intelligent Robots and Systems. IEEE, 2013.

[5] Paull, L., Seto, M., and Li, H., Area coverage planning that accounts for pose uncertainty with an AUV seabed surveying application, 2014 IEEE International Conference on Robotics and Automation (ICRA). IEEE, 2014.

[6] Mood, A., Introduction to the Theory of Statistics, pp. 200, 1950.

[7] P. Athanasios, Probability, random variables, and stochastic processes, pp. 141. McGrawHill, 1991.

[8] N. Metropolis, A. Rosenbluth, M. Rosenbluth, A. Teller, and E. Teller, Equation of state calculations by fast computing machines, J. Chem. Phys., vol. 21, no. 6, pp. 10871092, 1953. 